\title{
Effect of Preoperative Albumin on Post Operative Outcome Following Colorectal Surgeries
}

\section{Authors \\ Dr S.B.Ramya, Dr Rubina Singh, Prof. Arulappan.T, Dr Sivaraja, Dr Vikas}

\section{Introduction}

Albumin is a blood plasma protein synthesized in the liver. It is the single most abundant protein in plasma and constitutes about 2/3rd of the total protein content. Because it is the main protein in human blood, decreased synthesis or losses result in impaired regulation of intravascular oncotic pressure and manifests as edema. Serum albumin is a good and simple predictor of surgical risk and has a close correlation with the degree of malnutrition. A decrease in serum albumin concentrations is an almost inevitable findings in disease states, and is primarily mediated in the acute phase by alterations in vascular permeability and redistribution. This change is not disease specific but marked changes that persist are generally associated with poorer prognosis. Lower serum albumin levels showed a trend toward having higher post operative mortality rates and had significantly higher rates of several complications than did patients with higher serum albumin levels. Hypoalbuminemia is a predictor of delayed recovery of bowel function and is strongly associated with post operative complications after surgeries for colonic pathologies. The role of albumin in maintenance of homeostasis is well known but the mechanism of its deficiency and the harmful effects of the same in patients especially who have undergone major surgery have not been well understood till now. This study tries to determine the relationship between hypoalbuminemia and the incidence of complications and to evaluate the effects of hypoalbuminemia on complications namely wound infections, respiratory infections, UTI, anastomotic leaks, post operative bowel function and hospital stay of the patient.

\section{Aim of the study}

To determine the effect of preoperative albumin levels on postoperative outcome in patients undergoing elective surgery for benign and malignant colorectal diseases in Sri Ramachandra university between April 2014- December 2016.

\section{Materials and Methods}

This is a prospective study from april 2014december 2016 in Department of general surgery, Sri Ramachandra university. Patients who underwent colorectal surgery with resection and anastomosis constituted the population study. Patients were categorised into mild (3.1-3.5mg/dl) severe $(<3 \mathrm{mg} / \mathrm{dl})$ and normal albumin ranges $(>3.5 \mathrm{mg} / \mathrm{dl})$ Preoperative albumin levels were analysed interrelation to age, gender, disease pattern (benign/malignant) and the stage of disease in patients with malignancy. incidence of hypoalbuminemia in right and left colon diseases 
was studied .postoperative outcomes in terms of wound infections, anastomotic leaks, Respiratory tract infections, UTI, day of restoration of feeds , length of postoperative stay, and mortality in relation to albumin levels were analysed.

\section{Inclusion Criteria}

All patients above 18 years undergoing elective colorectal surgery for both benign and malignant diseases.

\section{Exclusion Criteria}

- Emergency surgery

- surgeries resulting in stomas.

\section{Discussion}

A total of 50 patients comprised the study population of which $9(18 \%)$ had severe hypoalbuminemia, $9(18 \%)$ had mild hypoaluminemia and the others 36(64\%) with normal albummin levels. In men the incidence of hypoalbuminemia was $38.5 \%$, in contrast to women who had $27 \%$ with hypoalbuminemia. $44.4 \%$ of the patients in the age group of less than 60 years and $55.6 \%$ of the patients above 60 years had severe hypoalbuminemia $(<3 \mathrm{mg} / \mathrm{dl}) .55 .6 \%$ and $44.4 \%$ of the patients had mild hypoalbuminemia in the age group less than 60 years and above 60 years. In addition to this most of the patients within the age group above 60 years had a higher incidence of malignancy in comparison to the younger age group who mostly presented with benign diseases Of the 50 patients assessed in the study ,39 patients had malignant pathology and the remaining 11 underwent resection and anastomosis for benign causes. Of the 39 patients, 2 patients had transverse colon growths and 16 patients had left colonic carcinomas .In benign diseases, it was noted that $27 \%$ had severe hypoalbuminemia and $9 \%$ had mild hypoalbuminemia. In malignant diseases, $15 \%$ and $21 \%$ had severe and mild hypoalbuminemia .on assessing the albumin levels in Right and Left colonic carcinoma patients, $25 \%$ had hypoalbumi-nemia whereas right colonic carcinoma patients $43 \%$ had hypoalbuminemia. Despite the significant discrepancy in the number of patients in each stage, it was noted that in stage :1, 33\% had hypoalbuminemia and in stage 4, 67\% had hypoalbuminemia $55.6 \%$ of patients with severe hypoalbuminemia and $44.4 \%$ of patients with mild hypoalbuminemia were found to have wound infections at the surgical site. This is significantly higher in comparison to only $18.8 \%$ of th patients in normal albumin range. Other studies i.e. Alves et al and Lohsiriwat et al showed incidence of wound infections to be $9.6 \%$ and $17 \%$ respectively .In our study none of the patients had anastomotic leaks, whereas Alves et al and Lohsiriwat et al documented $5 \%$ of which almost half underwent reexploration. and $4.4 \%$ respectively. Respiratory tract infections were noted to be $33.3 \%, 11.1 \%$ and $9.4 \%$ in the severe, mild hypoalbuminemia and normal albumin groups respectively. Respiratory infections was noted to be $1 \%$ by lohsiriwat et al, but the sample size was significantly larger in comparison to our study. The percentage of patients found to have UTI was found to be the highest i.e $22.2 \%$ in patients with mild hypoalbuminemia, followed by $11.1 \%$ in patients with severe hypoalbuminemia and $9.4 \%$ in patients with normal albumin levels. Unlike respiratory and wound infections, there was no correlation found between the albumin levels and the incidence of UTI, hence it could be due to other factors i.e. comorbidites or procedure related. Alves et al and Lohsiriwat et al showed incidence of UTI to be $10.7 \%$ and $1 \%$ In patients with severe hypoalbuminemia, only $11.1 \%$ of the patients were started on oral feeds within 3 days whereas in the normal albumin range $21.9 \%$ of the patients were started on feeds within 3 days. $22.2 \%$ with severe hypoalbuminemia were started on delayed oral feeds post surgery in comparison to $6.2 \%$ with normal albumin levels. Hence it was observed that patients with hypoalbuminemia were started on feeds at a later date possibly due to delay in resumption of bowel activity. Only $33.3 \%$ of the patients with severe hypoalbuminemia were discharged within 10 days 
of the procedure in contrast to significantly higher percentage of patients with mild hypoalbuminemia $(44.4 \%)$ and normal albumin level (56.3\%). Lohsiriwat et al also documented a significantly longer postoperative stay in hypoalbuminemic patients. In our study, the reason for prolonged hospital stay in patients with hypoalbuminemia was noted to be post opartive wound infection and respiratory infections.

\section{Conclusion}

In our study the age group of patients ranged from $20-81$ years, the majority of patients were around 50-60 years of age with a male preponderance. It was seen that severe hypoalbuminemia was noted in $55.6 \%$ of the patients who were above 60 years. $55.6 \%$ of patients with severe hypoalbuminemia $44.4 \%$ with mild hypoalbuminemia were found to have wound infections in comparison to $18.8 \%$ of the patients in the normal albumin range. Respiratory infections were noted to be $33.3 \%$, $11.1 \%$ and $9.4 \%$ in the severe, mild hypoalbuminemia and normal albumin groups respectively. $11.1 \%$ of the patients with severe hypoalbuminemia had urinary tract infections versus $9.4 \%$ in patients with normal albumin levels. The percentage of patients found to have UTI was found to be the highest i.e $22.2 \%$ in patients with mild hypoalbuminemia. In severe hypoalbuminemia, only $11.1 \%$ of the patients were started on feeds within three days of surgery whereas $21.9 \%$ of the patients with normal albumin levels were started on feeds within postoperative day three. $33.3 \%$ of the patients with severe hypoalbuminemia were discharged within 10 days of the procedure. In mild hypoalbuminemia, $44.4 \%$ and $56.3 \%$ of the patients with the normal albumin levels were being discharged. None of the patients in our study, due to ARDS inspite of normal albumin level. There was one mortality in our study, due to ARDS inspite of normal albumin levels. In conclusion, preoperative hypoalbuminemia is an independent risk factor for post operative complications following colorectal surgery.
Hypoalbuminemic patients had a higher incidence of infections, delayed postoperative bowel activity and longer post operative stay. Hence, low serum albumin may be used as a simple and low cost prognostic tool to predict the risk of adverse surgical outcomes. 